\title{
New mechanisms of vesicles migration
}

\author{
Viviana Aursulesei ${ }^{1}$, Decebal Vasincu ${ }^{2}$, Daniel Timofte ${ }^{3}$, Lucia Vrajitoriu ${ }^{4}$, Irina Gatu ${ }^{4}$, \\ Dan D. Iacob ${ }^{4}$, Vlad Ghizdovat ${ }^{2,4}$, Calin Buzea ${ }^{5}$ and Maricel Agop ${ }^{6,7}$ \\ 1 "Grigore T. Popa" University of Medicine and Pharmacy, Medical First Department, 16 University Str., Iaşi 700115, Romania \\ 2 "Grigore T. Popa" University of Medicine and Pharmacy, Biophysical Department, 16 University Str., Iaşi 700115, Romania \\ 3 "Grigore T. Popa" University of Medicine and Pharmacy, Surgery Department, 16 University Str., Iaşi 700115, Romania \\ ${ }^{4}$ Faculty of Physics, "Alexandru Ioan Cuza" University, Blvd. Carol I, no. 11, Iaşi 700506, Romania \\ ${ }^{5}$ National Institute of Research and Development for Technical Physics, D. Mangeron 47, Iaşi 700050, Romania \\ ${ }^{6}$ Lasers, Atoms and Molecules Physics Laboratory, University of Science and Technology, Villeneuve d'Ascq. 59655 Lille, France \\ ${ }^{7}$ Department of Physics, "Gh. Asachi" Technical University, Iasi 700050, Romania
}

\begin{abstract}
In multicellular organisms, both health and disease are defined by means of communication patterns involving the component cells. Despite the intricate networks of soluble mediators, cells are also programed to exchange complex messages pre-assembled as multimolecular cargo of membranous structures known as extracellular vesicles (EVs). Several biogenetic pathways produce EVs with different properties able to orchestrate neighboring cell reactions or to establish an environment ripe for spreading tumor cells. Such an effect is in fact an extension of similar physiological roles played by exosomes in guiding cell migration under nontumoral tissue remodeling and organogenesis. We start with a biological thought experiment equivalent to Bénard's experiment, involving a fluid layer of EVs adherent to an extracellular matrix, in a haptotactic gradient, then, we build and present the first Lorenz model for EVs migration. Using Galerkin's method of reducing a system of partial differential equations to a system of ordinary differential equations, a biological Lorenz system is developed. Such a physical frame distributing individual molecular or exosomal type cell-guiding cues in the extracellular matrix space could serve as a guide for tissue neoformation of the budding pattern in nontumoral or tumoral instances.
\end{abstract}

Key words: Extracellular vesicles - Extracellular matrix space - Cell-guiding cues - Budding pattern - Bénard-Rayleigh model - Biological Lorenz system

\section{Introduction}

Communication patterns play a vital role in multicellular organisms because they define the healthy and diseased states of these organisms, through communication patterns involving the component cells. Despite the fact that soluble mediators form intricate networks, cells are also programed to transmit complex messages, which are pre-assembled as a multimolecular cargo of membranous structures named "extracellular vesicles" (EVs). EVs with various properties are produced by an array of biogenetic pathways. These

Correspondence to: Maricel Agop, Department of Physics, "Gh. Asachi” Technical University, Iasi 700050, Romania E-mail: m.agop@yahoo.com
EVs can induce neighboring cell reactions or establish an environment favorable for tumor cells and their uncontrolled expansion. This effect is actually an extension of similar physiological roles plated by exosomes in guiding cell migration under nontumoral tissue remodeling and organogenesis. Accordingly, there is increasing support that vesicle trafficking, including the release of EVs, is a highly important process in tumorigenesis and embryogenesis (Helfrich 1973; Lipowsky and Sackmann 1995; Kraus et al. 1996; Prost and Bruinsma 1996; Durand et al. 1997; Cantat and Misbah 1999a,b; Kern and Fourcade 1999; Seifert 1999; Sukumuran and Seifert 2001).

In the field of cell's biology, we call vesicles those small bags wrapped in a membrane forming part of eukaryotic cell organelles. These sacs help to transport and absorb 
proteins, enzymes or meet other needs of the cell. Inside the membrane bag of a vesicle, there are macromolecules which require the ability to move outside the cell walls. The membrane encircling the bag merges with the outer wall of the cell to allow such macromolecules to pass through the wall. The vesicles are important parts of the human cells, although they are also found in other multicellular organisms.

Cells found in humans, plants and animals use a variety of types of vesicles, depending on the type of cell and its specific intended function. For example, one type of vesicles, lysosomes, are necessary for the process of digestion. Lysosomes contain enzymes that breakdown food cells. With food absorption, a lysosome vesicle bonds to the food holding cell and releases enzymes by a process called phagocytosis. These enzymes break down food cells into smaller parts that can be better absorbed by other cells.

Secretory vesicles are frequently associated with nerve cells in humans or animals. Their membranes sacs contain neurotransmitters. Nervous system through hormonal signals triggers these components. Through the process of exocytosis, the secretory vesicle's outer membrane adheres to the nerve terminal and releases neurotransmitters in the area of the nerve endings, known as the synaptic cleft. Neurotransmitters carry information from one nerve terminal to the next, across the entire central nervous system, way up to the brain.

Vesicles, in their role as cellular mechanism are internally appointed for transport, uptake and storage of numerous imperative bodily functions. Without these tiny bags wrapped in membranes, cells could not make the exchange of materials necessary to maintain their healthy development and other crucial processes. In short, with no vesicles, humans

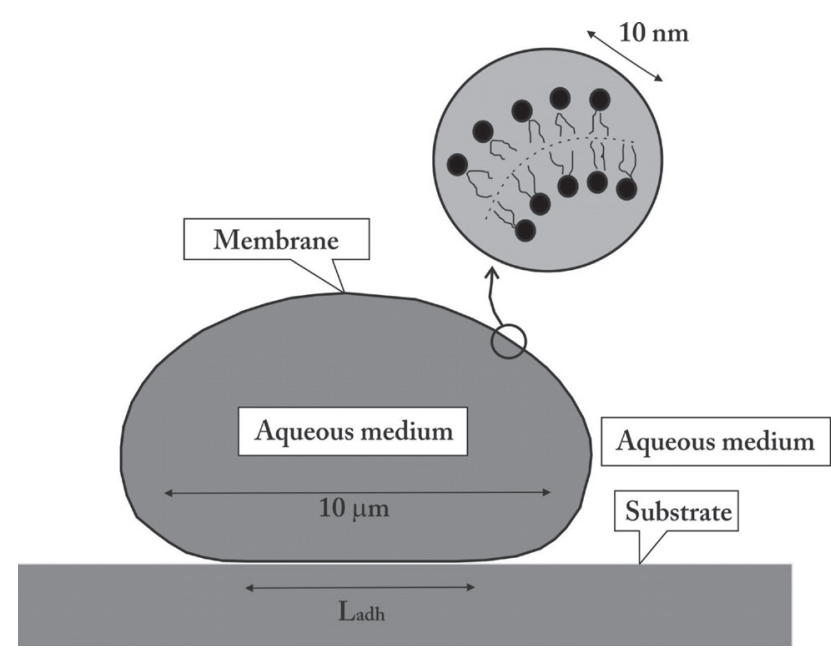

Figure 1. Schematic view of a vesicle emphasizing its microscopic structure: a bilayer of phospholipidic molecules. $\mathrm{L}_{\mathrm{adh}}$, the adherence length of the membrane. and other multicellular organisms could not exist, because the essential cellular chemical processes would have no other method to exchange key materials.

Since there is increasing support that vesicle trafficking, including the release of EVs, is a highly important process in tumorigenesis, embryogenesis and tissue remodeling, in this work we present an extensive discussion on the EVs convection in haptotaxis with hydrodynamical dissipation (i.e. a novel mechanism for vesicle migration).

\section{Materials and Methods}

Vesicles are closed membranes floating in an aqueous solution (see Fig. 1). These membranes serve as an efficient permeability barrier. The vesicles mimic one of the most primitive and mechanically flexible dividing interfaces between the inside and the outside of a cell. Generally, the fluid enclosed by the membrane is incompressible in order that the vesicle evolves at a constant volume. Moreover, the membrane exchanges no phospholipid molecules with the solution; as a result its area remaining constant as time passes. Helfrich (1973) described very well the vesicle's bending energy in its equilibrium state, which is compatible with the constraints above, i.e. constant volume and area. Even if the model is relatively simple it produces a variety of equilibrium profiles, such as, discocytes (bearing resemblance with red blood cells), stomatocytes, as well as forms presenting higher topologies (such as n-genus torus) that have been also observed experimentally (Lipowsky and Sackmann 1995). We identify works studying alignments of vesicle in shear flows (Kraus et al. 1996), fluctuations out of equilibrium (Prost and Bruinsma 1996), lift forces (Cantat and Misbah 1999; Seifert 1999, Sukumuran and Seifert 2001), migration of vesicle in the proximity of a substrate (Durand et al. 1997; Cantat and Misbah 1999) or in gravity fields (Kern and Fourcade 1999) and also vesicle tumbling (Biben and Misbah 2002). One may note several recent experiments dealing with vesicle migration (Nardi et al. 1999; Lortz et al. 2000; Abrakarian et al. 2001; Abrakarian et al. 2002).

Considering the vesicle migration, we acknowledge it involves hydrodynamical dissipation in the neighboring fluid as well as inside the vesicle, and, in principle, between the two mono-layers which may glide with respect to each other. Furthermore, during motion on the substrate the dynamics of a vesicle may be restricted not only by the hydrodynamical flow but also by mechanisms of breaking and restoring of bonds on the substrate. It is obvious that the slowest mechanism limits the motion. Here we focus on a situation where hydrodynamics are the limiting factors and we give out dissipation associated with bonds on the substrate.

We consider a vesicle which is initially adhering on a flat surface. An adhesion gradient is considered along the 
substrate. The vesicle moves in the direction of increasing adhesion energy (see Fig. 2) - it is named haptotaxis (a motion induced by an adhesion gradient).

A highly permeable vesicle can be pulled in a fluid without any resistance (and without an inner area change), whereas an impermeable one would feel a drag force. The assumption of local impermeability is legitimate. This entails that the fluid velocity at the membrane is equal that of the membrane itself (Cantat et al. 2003).

On a vesicle's scale $(\mathrm{R} \sim 10 \mu \mathrm{m})$ and for the expected velocities $(\mathrm{V} \sim 1 \mu \mathrm{m} / \mathrm{s})$, dynamics are fully dominated by dissipative processes. The energy added is instantly dissipated in various degrees of freedom. Local dissipation due to molecular reorganization, characterized by Leslie's coefficient, is negligible in comparison to hydrodynamics modes (Brochard and Lennon 1975).

If dissipation is dominated by bulk effects, as shown in Landau and Lifshitz (1987) we are in the position to write down the basic governing equations for convective vesicles in a geometry depicted in Fig. 3, since it was proved that the velocity field obeys the Stokes equations (Cantat et al. 2003).

In an original atmospheric system, the non-even distribution of ascending water droplets is determined by the interplay between solar energy-induced thermal gradients, thermal diffusivity, friction, and gravity. Ultimately, the mathematics of this model shapes the umbrella-like or budding appearance of structures like cumulonimbus clouds. This model can better or uniquely describe those types of structural dynamics not explained under fractal, simple/ linear and several other types of models.

Acknowledging that similar patterns occur in various biological spaces, we think that the same mathematical determinism can be ascribed. Thus, some histoarchitectural prototypic structures, like the capillary sprouting, embryologic organ, or even tumor buds of some types of cancer lesions might be in fact sculpted in that shape because gradients of molecular cues called morphogens can be deployed

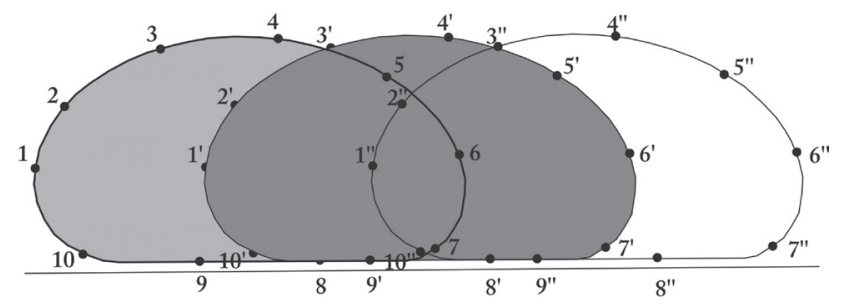

Figure 2. Stationary vesicle profiles are depicted. The vesicle is moving from the left (smaller adhesion) to the right (stronger adhesion); a few discretization points are represented and the arrow allows following one of these at three successive times. One can observe here the rolling and sliding components of the vesicle's motion. within the same manner water droplets can organize within nascent clouds.

Assuming that this organization also applies in biological systems, and that the EVs release can be considered among various processes organizing the budding tissue pattern, we think that the Lorenz model (Lorenz 1963, 2005) can govern their dynamics too. EVs would be particularly interesting as controllers of the tissue shape specification because they can include enzymatically active components (not found in conventional molecular morphogens), and thus might actively interact with the extracellular matrix (ECM) fibers within their migration. Deployment of certain matrix degrading enzymes (MDEs) by EVs can modify this space while diffusing (event not produced by simple morphogens, attractive chemokines or repulsive semaphorins). This activity changes the topography of the ECM and creates spatial gradients directing the migration of subsequent EVs by haptotaxis - a mechanism better described for cell migration.

Let us consider the following thought biological experiment, equivalent to the Bénard experiment: a fluid layer of extracellular vesicles adherent on an ECM, in a haptotactic gradient. The fluid layer presents an unstable stratification of the potential density in a field of forces: the dense fluid is placed in front of the less dense one. We assume that in

\section{$\mathrm{C}_{1}$}
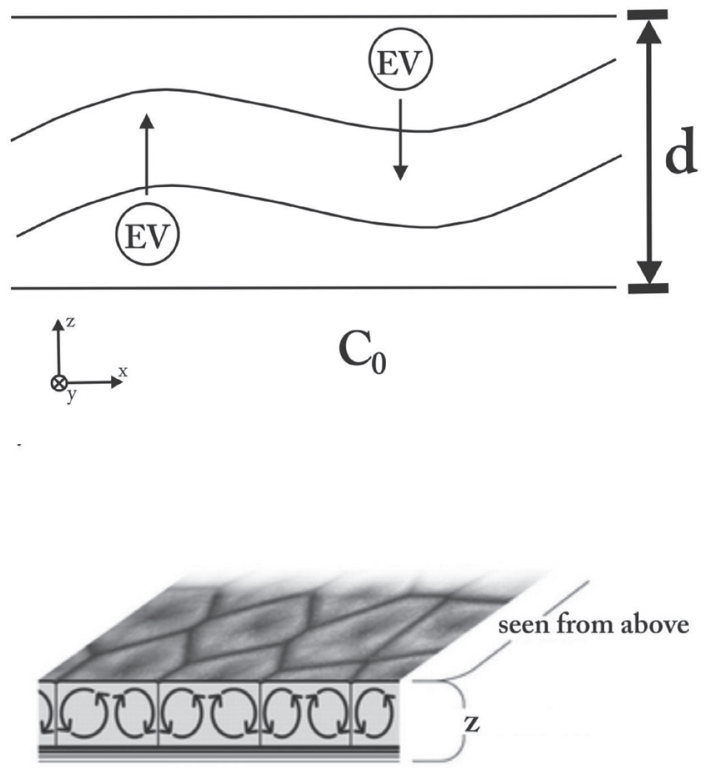

Figure 3. Convective extracellular vesicles (EVs) geometry. A fluid layer of thickness $\mathrm{d}$ of EVs, adherent on an extracellular matrix (ECM), is subjected to a gradient of concentration, where $\Delta \mathrm{C}=\mathrm{C} 1$ $-\mathrm{C} 0>0$ is the difference of concentration between the front and back boundaries of the fluid layer. 
the basic state the layer of fluid of thickness $d$ is subjected to a gradient of concentration

$$
\beta=\frac{C_{1}-C_{0}}{d}>0
$$

$\Delta \mathrm{C}=\mathrm{C}_{1}-\mathrm{C}_{0}>0$ is the difference of concentration between the front and back boundaries of the fluid layer. The regime with the fluid at rest and a non-perturbed distribution of concentration, belongs to the thermodynamic branch, which is continuously linking the non-equilibrium stationary state $(\Delta \mathrm{C} \neq 0)$ with the equilibrium state $(\Delta \mathrm{C}=0)$ (Fig. 3).

We examine the evolution of a concentration fluctuation $\theta$ around the non-perturbed concentration profile $C_{0}(z)$.

Two dissipative processes tend to maintain the fluid at rest:

- friction (motion amortization through viscosity);

- ECM degradation subsequent to MDE's activity allowing vesicle trespassing - which lowers the concentration of the ECM, thus diminishing the forward, or advancing force.

The instability cannot be developed unless the EV is accelerated enough to overcome the effect of these dissipative processes. The gradient of concentration $\beta$ which is the control parameter of this instability has to surpass a critical value $\beta_{C}$. Over this critical value, an organized structure of convection cells may appear.

For a one component fluid, the mass, momentum and internal energy equations are (Lorenz 1963)

$$
\begin{aligned}
& \frac{\partial \rho}{\partial t}+\nabla \cdot(\rho \mathbf{v})=0 \\
& \frac{\partial(\rho \mathbf{v})}{\partial t}+\nabla \cdot(\vec{\Pi}+\rho \mathbf{v} \mathbf{v})=\rho \mathbf{g} \\
& \frac{\partial(\rho \varepsilon)}{\partial t}+\nabla \cdot\left(\rho \varepsilon \mathbf{v}+\mathbf{j}_{d}\right)=-\vec{\Pi} \otimes(\nabla \mathbf{v})
\end{aligned}
$$

where $\rho$ represents the mass density of the fluid, $v$ its speed, $\boldsymbol{g}$ acceleration of a field of forces, $\varepsilon$ the internal energy of the unit volume, and $\boldsymbol{j}_{d}$ the flux of ECM degraded by signals received from EVs. Here $\vec{\Pi}$ is the stress tensor and $\otimes$ denotes the product of two tensors,

$$
\vec{A} \otimes \vec{B}=A_{i j} B_{j i}
$$

and we use Einstein's summation convention (implicit sum over repeating indices). The stress tensor can be written

$$
\vec{\Pi}=\vec{\Pi}^{e}+\vec{\Pi}^{v}
$$

$\vec{\Pi}^{e}$ is the equilibrium part and depends on the state of the system. $\vec{\Pi}^{v}$ represents the non-equilibrium part and is named viscous stress tensor. At equilibrium, this part vanishes. For an isotropic medium at rest,

$$
\vec{\Pi}^{e}=\left(\begin{array}{ccc}
p & 0 & 0 \\
0 & p & 0 \\
0 & 0 & p
\end{array}\right)=p \vec{I}
$$

where $p$ is the hydrostatic pressure. For viscous systems at non-equilibrium, the viscous stress tensor is not null. According to equation (3) and equation (4), the stress tensor will be, for homogeneous and isotropic viscous systems, at non-equilibrium

$$
\vec{\Pi}=p \vec{I}+\vec{\Pi}^{v}
$$

We start with the following assumptions:

a) the fluid is Newtonian; as a result the stress tensor is given by equation (5), where the viscous stress tensor is (Lorenz 1963)

$$
\Pi_{\alpha \beta}^{v}=-\eta\left(\frac{\partial v_{\alpha}}{\partial x_{\beta}}+\frac{\partial v_{\beta}}{\partial x_{\alpha}}-\frac{2}{3} \delta_{\alpha \beta}(\nabla \cdot \mathbf{v})\right)-\zeta \delta_{\alpha \beta}(\nabla \cdot \mathbf{v})
$$

with coefficients $\eta$ and $\zeta$ independent of velocity, the tangential (shear) and bulk viscosity, respectively;

b) ECM degrading by MDE's from EVs is described by the Fourier differential equation

$$
\mathbf{j}_{d}=-\lambda \nabla C
$$

where $\lambda$ is the haptotactic coefficient. Let us note that this equation is an alternative form (biological form) of the standard Fourier's law (Fritz 1991), which states that the time rate of heat transfer through a material is proportional to the negative gradient in the temperature and to the area, at right angles to that gradient, through which the heat flows.

c) haptotactic energy expansion is linear

$$
\delta \rho=\rho-\rho_{0}=-\rho_{0} \alpha \varepsilon=-\rho_{0} \alpha k_{h}\left(C-C_{0}\right)=-\rho_{0} \chi\left(C-C_{0}\right)
$$

where we used the expression of the haptotactic energy $\varepsilon=$ $k_{h}\left(C-C_{0}\right), k_{h}$ being the haptotactic energy constant. In Eq. (7), $\alpha$ is the haptotactic energy expansion constant and $\chi=\alpha k_{h}$ is the haptotactic expansion constant;

d) the fluid satisfies a state equation: consequently, its internal energy is (up to a constant factor)

$\varepsilon=k_{b} C$

where $k_{b}$ is the state constant;

e) in most liquids, thermal expansion is small. We choose everywhere a constant density, denoted by $\rho_{0}$, except the momentum equation. 
With these approximations, the system of Eqs. $(2 a-c)$ leads to the Boussinesq type system of equations (Manneville 1991; Schuster 1995)

$$
\begin{aligned}
& \nabla \cdot \mathbf{v}=0 \\
& \rho_{0}\left[\frac{\partial \mathbf{v}}{\partial t}+(\mathbf{v} \cdot \nabla) \mathbf{v}\right]+\nabla p=\left(\rho_{0}+\delta \rho\right) \mathbf{g}+\eta \nabla^{2} \mathbf{v} \\
& \frac{\partial C}{\partial t}+(\mathbf{v} \cdot \nabla) C=\frac{\lambda}{\rho_{0} k_{b}} \nabla^{2} C
\end{aligned}
$$

where $\rho$ is the perturbed density

$$
\rho=\rho_{0}+\delta \rho
$$

Eq. (9a) represents the incompressibility condition for the fluid. Let us note that the Boussinesq system of equation has been used for the first time for water waves, taking into account the vertical structure of the horizontal and vertical flow velocity. This resulted in non-linear partial differential equations, called Boussinesq-type equations, which incorporate frequency dispersion (as opposite to the shallow water equations, which are not frequency-dispersive) (Boussinesq 1871; Johnson 1997).

Convection occurs in the fluid layer when the forward, or advancing force, resulted from energy expansion, surpasses the viscous forces. We may define now a Rayleigh type number

$$
R=\frac{\left|\mathbf{F}_{\text {asc }}\right|}{\left|\mathbf{F}_{\text {visc }}\right|} \approx \frac{\left|\frac{\delta \rho \mathbf{g}}{\rho_{0}}\right|}{\left|\frac{\eta \nabla^{2} \mathbf{v}}{\rho_{0}}\right|}
$$

The density perturbation satisfies, according to Eq. (7)

$$
\frac{\delta \rho}{\rho_{0}} \approx \chi \Delta C
$$

On the other side, from the internal energy equation Eq. (9c), it results

$$
v \approx \frac{\lambda}{\rho_{0} k_{b}} \frac{1}{d}
$$

Replacing Eqs. (12) and (13) in Eq. (11), and taking into account Eq. (1), we get a biological Rayleigh number

$$
R=\frac{\chi \beta \rho_{0} k_{b} g}{v \lambda} d^{4}
$$

where $v=\eta / \rho_{0}$ is the cinematic viscosity. For the Bénard convection, the biological Rayleigh number plays the part of a control parameter. The convection occurs for $R>R_{\text {critical. }}$. Most of the time, $R$ is controlled by $\beta$, the gradient of concentration.
Within a biological context, $g$ can be specified by polar/ linear topography of semaphorins or/and chemokines, signals typically creating stable gradients to which EVs can respond.

We choose as reference state the rest stationary state $\left(v_{S}=0\right)$, for which the last two equations in the system of Eqs. $(9 a-c)$ reduce to

$$
\begin{aligned}
& \nabla p_{S}=-\rho_{S} g \hat{z}=-\rho_{0}\left[1-\chi\left(C_{S}-C_{0}\right)\right] g \hat{z} \\
& \nabla^{2} C_{S}=0
\end{aligned}
$$

where $\hat{z}$ is the versor of the vertical direction. We assume pressure and concentration varies only along the vertical direction, due to the geometry of the experiment. For concentration, the boundary conditions read $C(x, y, 0)=C_{0} ; C(x, y, d)=C_{1}$. Integrating Eq. (15b) with these boundary conditions, it results that, in the stationary reference state, the profile of the concentration in the vertical direction is linear

$$
C_{S}=C_{0}-\beta z
$$

with $\beta$, the gradient of concentration. Replacing Eq. (16) in Eq. (15a) and integrating, we get

$$
p_{S}(z)=p_{0}-\rho_{0} g\left(1+\frac{\chi \beta z}{2}\right) z
$$

The characteristics of the system in this state are independent of the kinetic coefficients $\eta$ and $\lambda$ which appear in Eqs. $(9 a-c)$. We study the stability of the reference state using the small perturbations method. The perturbed state is characterized by

$$
\begin{aligned}
& C=C_{S}(z)+\theta(\mathbf{r}, t) \\
& \rho=\rho_{S}(z)+\delta \rho(\mathbf{r}, t) \\
& p=p_{S}(z)+\delta p(\mathbf{r}, t) \\
& \mathbf{v}=\delta \mathbf{v}(\mathbf{r}, t)=(u, v, w)
\end{aligned}
$$

As can be seen from Eqs. (18a-d), the perturbations are functions of coordinate and time. Replacing Eqs. (18a-d) in the evolution equations of the Boussinesq approximation equations (Boussinesq 1871; Johnson 1997) (9a-c) and taking into account Eq. (16) and Eq. (17), we get, in the linear approximation, the following equations for the perturbations

$$
\begin{aligned}
& \nabla \cdot \delta \mathbf{v}=0 \\
& \frac{\partial \delta \mathbf{v}}{\partial t}=-\frac{1}{\rho_{0}} \nabla \delta p+\nu \nabla^{2} \delta \mathbf{v}+g \chi \theta \hat{z} \\
& \frac{\partial \theta}{\partial t}=\beta w+K \nabla^{2} \theta
\end{aligned}
$$


where $K=\frac{\lambda}{\rho_{0} k_{b}}$ is a coefficient. We pass to non-dimensional variables in Eqs. $(19 \mathrm{a}-\mathrm{c})$, using the transformations: $\mathbf{r}^{\prime}=\frac{\mathbf{r}}{d} ; t^{\prime}=\frac{t}{d^{2} / K} ; \theta^{\prime}=\frac{\theta}{\left(\frac{v K}{g \chi d^{3}}\right)} ; \delta \mathbf{v}^{\prime}=\frac{\delta \mathbf{v}}{K / d}$ and $\delta p^{\prime}=\frac{\delta p}{\left(\frac{\rho_{0} v K}{d^{2}}\right)}$. In these transformations, each parameter is reported to the corresponding characteristic value. We replace these transformations and give up, for simplicity, at the primes. The non-dimensional perturbations satisfy the equations

$$
\begin{aligned}
& \frac{1}{\operatorname{Pr}}\left(\frac{\partial \mathbf{v}}{\partial t}+\mathbf{v} \cdot \nabla \mathbf{v}\right)=-\nabla p+\theta \hat{z}+\nabla^{2} \mathbf{v} \\
& \frac{\partial \theta}{\partial t}+(\mathbf{v} \cdot \nabla) \theta=R w+\nabla^{2} \theta \\
& \nabla \cdot \mathbf{v}=0
\end{aligned}
$$

where $\operatorname{Pr}=v / K$ is the biological Prandtl number, and $R$ the biological Rayleigh number.

Beyond the instability threshold $\left(R>R_{C}\right)$, the reference state becomes unstable, with the occurrence of convection rolls. We assume they are parallel, thus the speed vector will always be perpendicular on their axis. We presume the rolls are parallel to the $O y$ axis; consequently, the speed component along this direction is null. The incompressibility condition becomes

$$
u_{x}+w_{z}=0
$$

Eq. (21) is satisfied if

$$
\begin{aligned}
& u=-\psi_{z} \\
& w=\psi_{x}
\end{aligned}
$$

where $\psi(x, y, z)$ is called the Lagrange stream function. The velocity field must satisfy the conditions imposed on the inferior and superior surfaces

$$
w_{z= \pm 1 / 2}=0
$$

If the surfaces are considered free (superficial tension forces are ignored), the tangential (shear) component of the stress tensor vanishes

$$
-\eta \frac{\partial \eta}{\partial z}_{\mid z= \pm 1 / 2}=0 \Rightarrow \frac{\partial u}{\partial z}_{\mid z= \pm 1 / 2}=0
$$

Using the Lagrange stream function, the limit conditions of Eq. (23) and Eq. (24) become

$$
\begin{aligned}
& \psi_{x \mid z= \pm 1 / 2}=0 \\
& \psi_{z z \mid z= \pm 1 / 2}=0
\end{aligned}
$$

We choose for $\psi$ the form

$$
\psi(x, z, t)=\psi_{1}(t) \cos (\pi z) \sin (q x)
$$

According to Eqs. (22a,b), the components of the velocity field are

$$
\begin{aligned}
& u=\pi \psi_{1}(t) \sin (\pi z) \sin (q x) \\
& w=q \psi_{1}(t) \cos (\pi z) \cos (q x)
\end{aligned}
$$

The momentum conservation Eq. (20a) for $x$ and $z$ directions becomes

$$
\begin{aligned}
& \frac{1}{\operatorname{Pr}}\left(u_{t}+u u_{x}+w u_{z}\right)=-p_{x}+\nabla^{2} u \\
& \frac{1}{\operatorname{Pr}}\left(w_{t}+u w_{x}+w w_{z}\right)=-p_{z}+\nabla^{2} w+\theta
\end{aligned}
$$

We derive Eq. (25a) with respect to $z$ and Eq. (25b) with respect to $x$. We get

$$
\begin{aligned}
& \frac{1}{\operatorname{Pr}}\left[u_{t z}+\frac{\partial}{\partial z}\left(u u_{x}+w u_{z}\right)\right]=-p_{x z}+\frac{\partial}{\partial z}\left(\nabla^{2} u\right) \\
& \frac{1}{\operatorname{Pr}}\left[w_{t x}+\frac{\partial}{\partial x}\left(u w_{x}+w w_{z}\right)\right]=-p_{z x}+\frac{\partial}{\partial z}\left(\nabla^{2} w\right)+\frac{\partial \theta}{\partial x}
\end{aligned}
$$

Adding these two equations leads to

$$
\begin{aligned}
& \frac{1}{\operatorname{Pr}}\left[-\left(\nabla^{2} \psi\right)_{t}+\frac{\partial}{\partial z}\left(u u_{x}+w u_{z}\right)-\frac{\partial}{\partial x}\left(u w_{x}+w w_{z}\right)\right]= \\
& =-\left(\nabla^{2} \psi\right)^{2}-\theta_{x}
\end{aligned}
$$

The concentration being fixed on the two borders, we have

$$
\theta_{\mid z= \pm 1 / 2}=0
$$

We choose for $\theta$ the form

$$
\theta(x, z, t)=\theta_{1}(t) \cos (\pi z) \cos (q x)+\theta_{2}(t) \sin (2 \pi z)
$$

Replacing in Eq. (26) the expressions for $u, w, \theta$ and $\psi$, we get

$$
\operatorname{Pr}^{-1} \dot{\psi}_{1}=\frac{q \theta_{1}}{\pi^{2}+q^{2}}-\left(\pi^{2}+q^{2}\right) \psi_{1}
$$

The internal energy equation becomes 


$$
\begin{aligned}
& \dot{\theta}_{1}=-\pi q \psi_{1} \theta_{2}+q R \psi_{1}-\left(\pi^{2}+q^{2}\right) \theta_{1} \\
& \dot{\theta}_{2}=\frac{1}{2} \pi q \psi_{1} \theta_{1}-4 \pi^{2} \theta_{2}
\end{aligned}
$$

$$
\begin{aligned}
& \dot{X}=\operatorname{Pr}(Y-X) \\
& \dot{Y}=-X Z+r X-Y \\
& \dot{Z}=X Y-b Z
\end{aligned}
$$

In the equations of evolution for amplitudes, Eq. (28) and where

Eqs. (29a,b), we make the change of variables

$$
\begin{aligned}
& t^{\prime}=\left(\pi^{2}+q^{2}\right) t \\
& X=\frac{\pi q}{\sqrt{2}\left(\pi^{2}+q^{2}\right)} \psi_{1} \\
& Y=\frac{\pi q^{2}}{\sqrt{2}\left(\pi^{2}+q^{2}\right)^{3}} \theta_{1} \\
& Z=\frac{\pi q^{2}}{\left(\pi^{2}+q^{2}\right)^{3}} \theta_{2}
\end{aligned}
$$

$r=\frac{q^{2}}{\left(\pi^{2}+q^{2}\right)^{3}} R$ and $b=\frac{4 \pi^{2}}{\pi^{2}+q^{2}}$

We recognize the system of equations (30) as the biological Lorenz system. The standard Lorenz systems and its fundamental properties are presented in Appendix A.

In Figs. 4-10, the trajectories, time evolutions, phase „portraits" and Fourier transforms for different parameter values of the biological Lorenz system are given. It results that, by increasing the $r$ parameter, a complex chaotic regimes

Thus we get the system of evolution equations
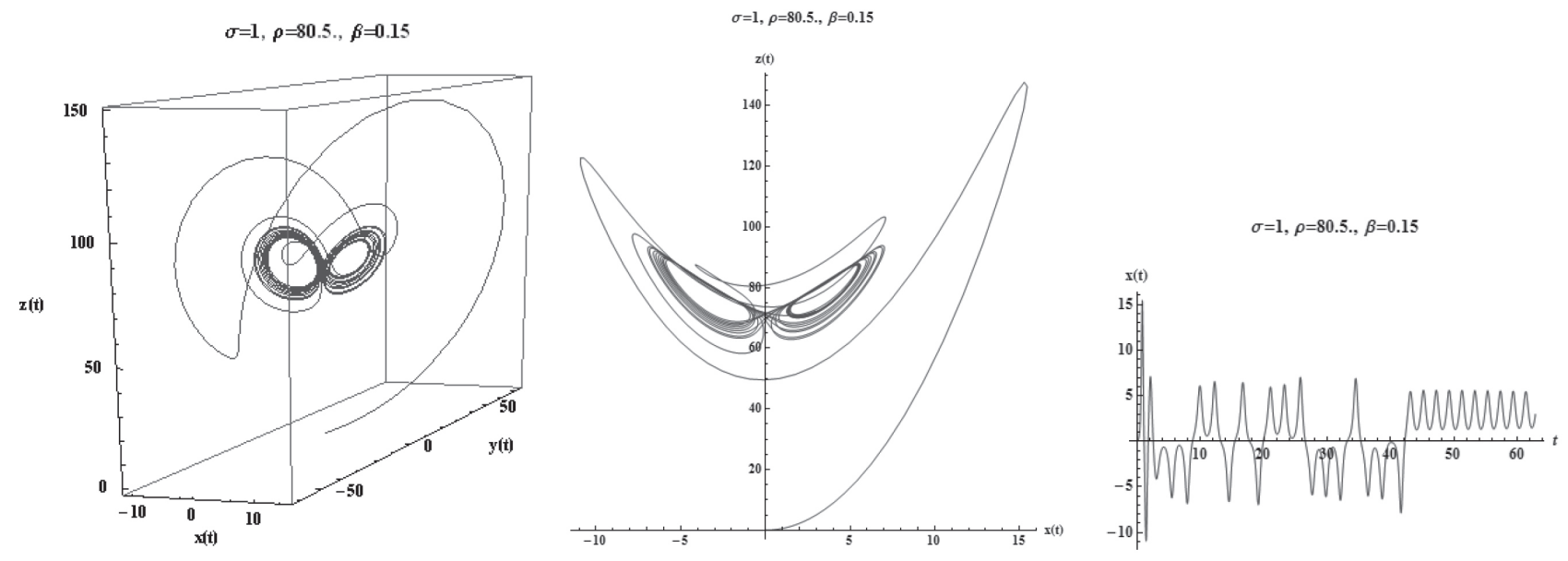

Figure 4. $3 \mathrm{D}, 2 \mathrm{D}$ phase pattern and time evolution for $\mathrm{r}=80, \mathrm{~b}=0.15$.
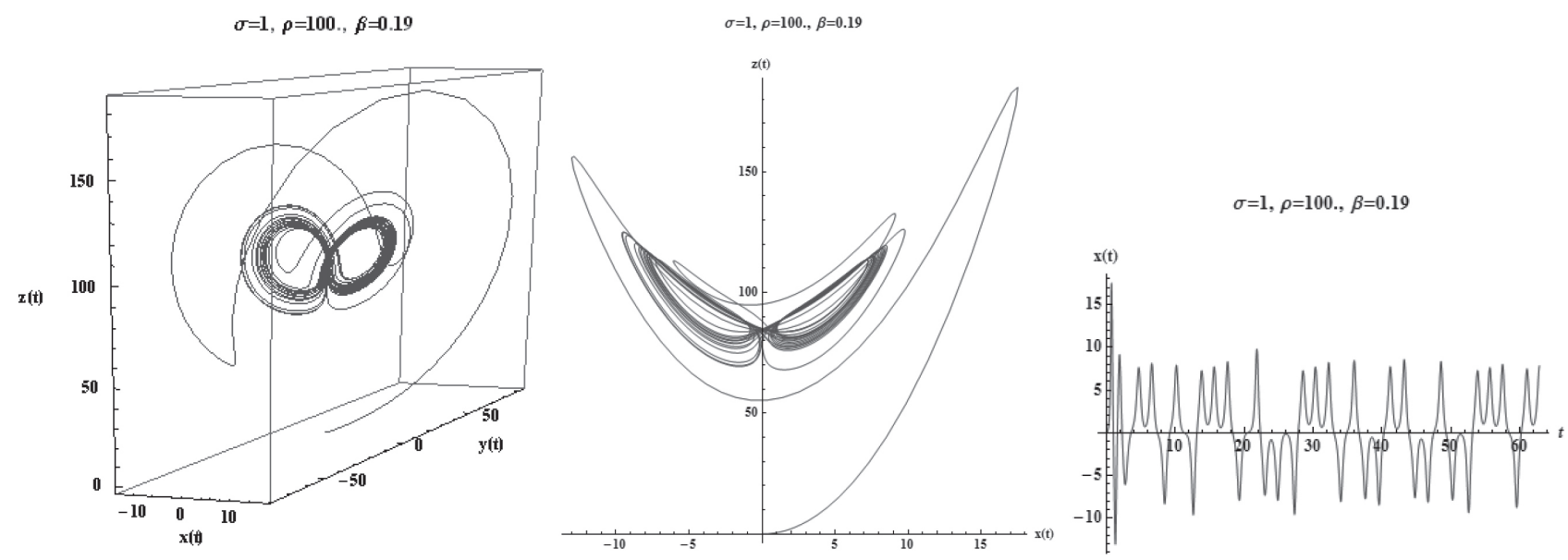

Figure 5. 3D, 2D phase pattern and time evolution for $r=100, b=0.19$. 

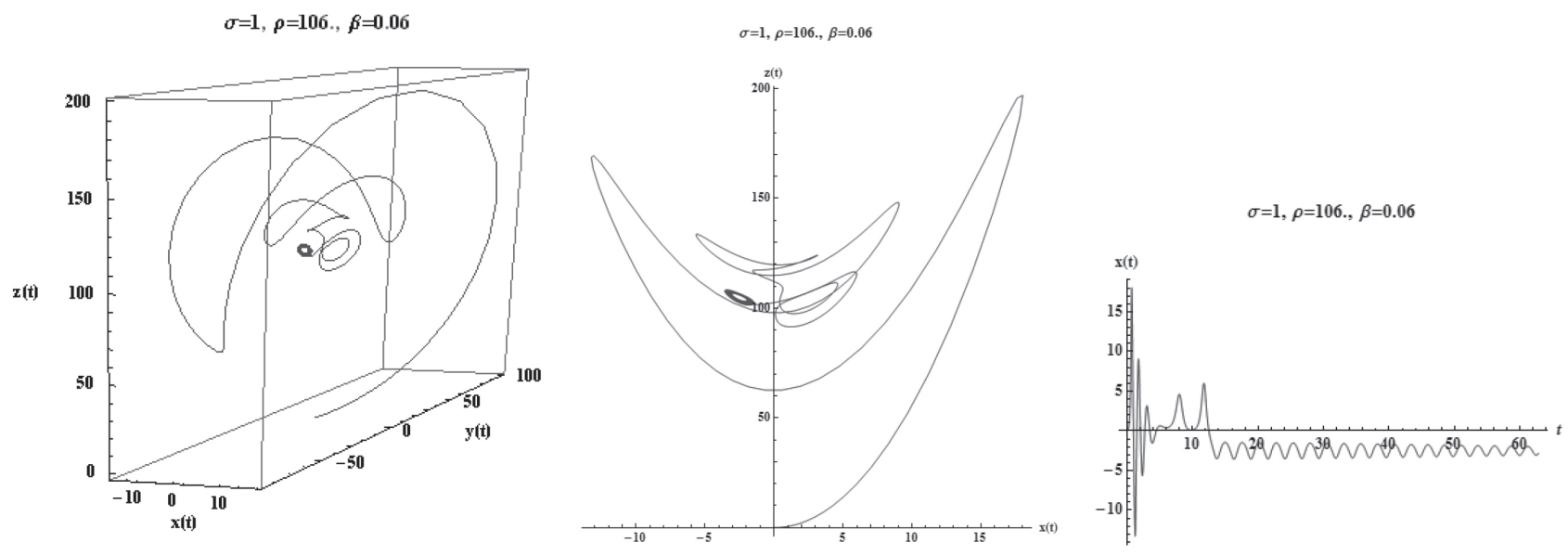

Figure 6. 3D, 2D phase pattern and time evolution for $r=106, b=0.06$.
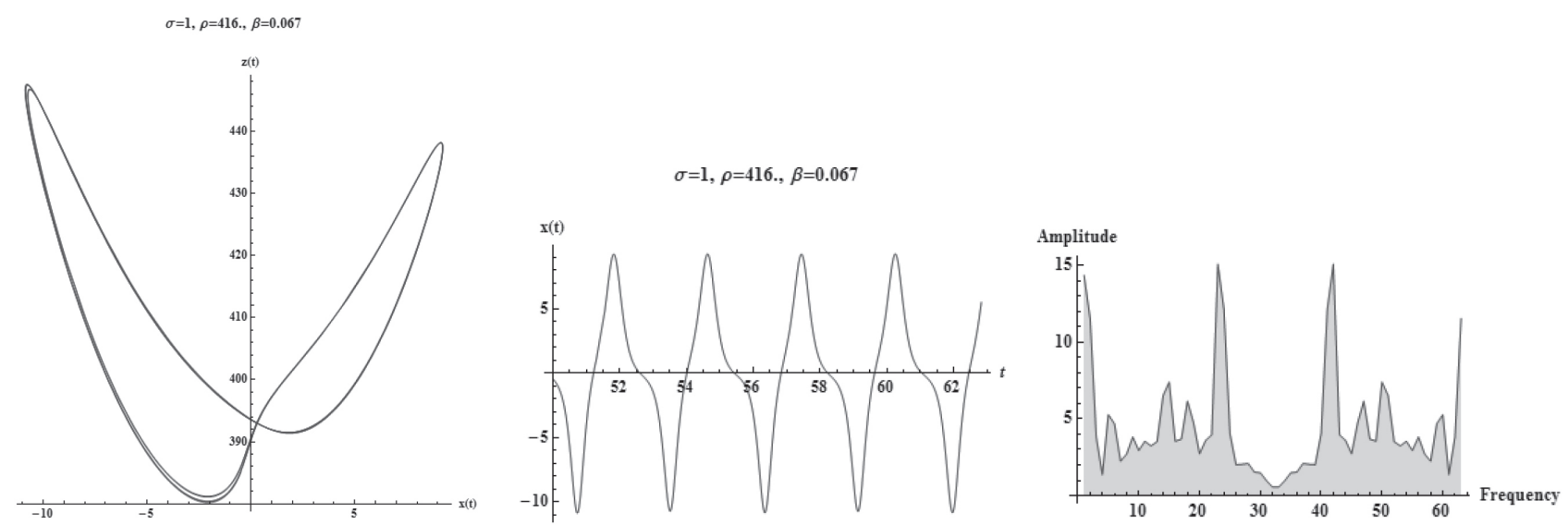

Figure 7. 2D phase "portrait", time evolution and Fourier transform for $r=416, b=0.067$.
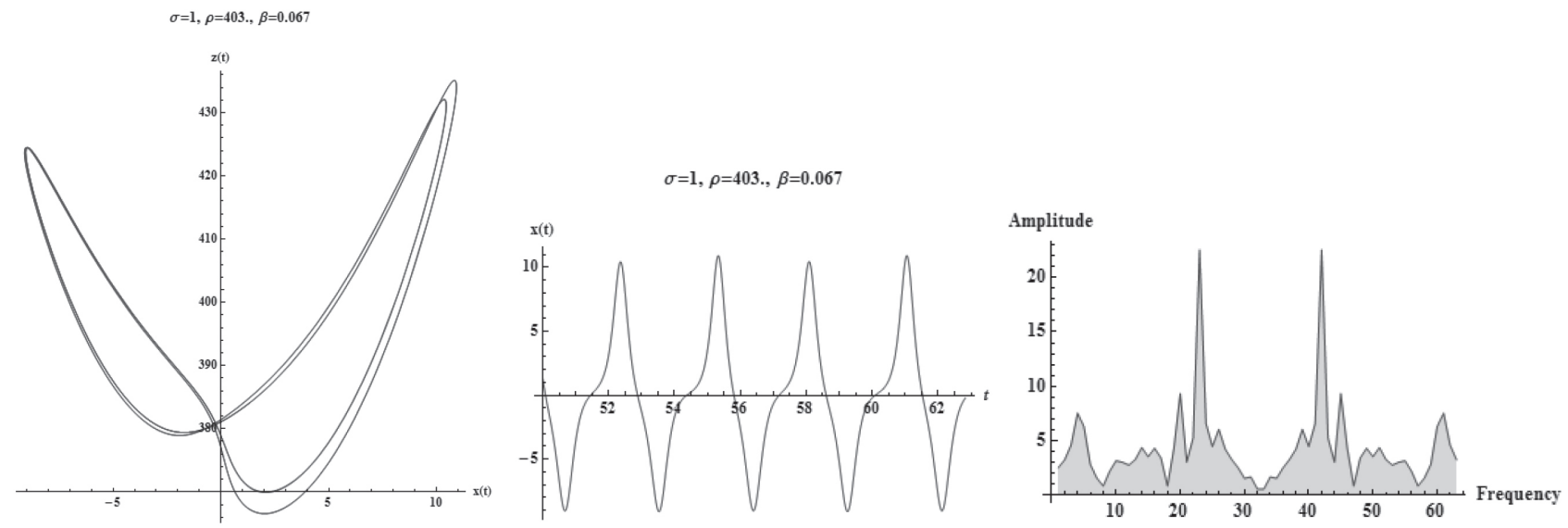

Figure 8. 2D phase "portrait", time evolution and Fourier transform for for $r=403, b=0.067$. 

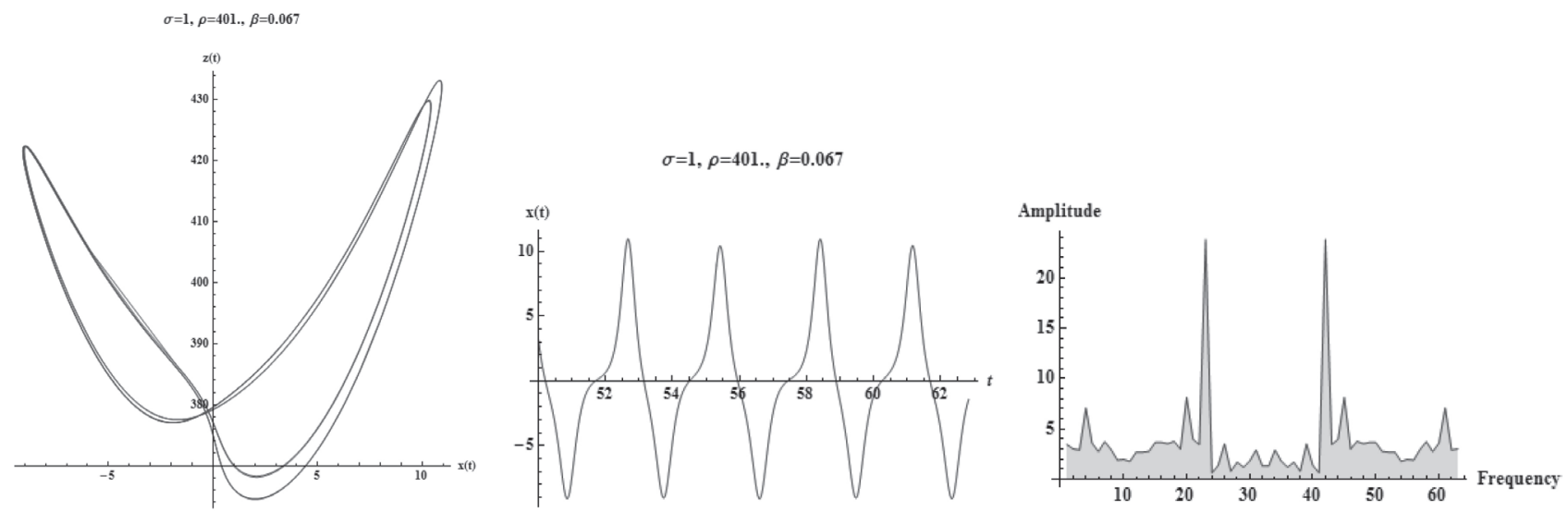

Figure 9. 2D phase "portrait", time evolution and Fourier transform for $r=401, \mathrm{~b}=0.067$.
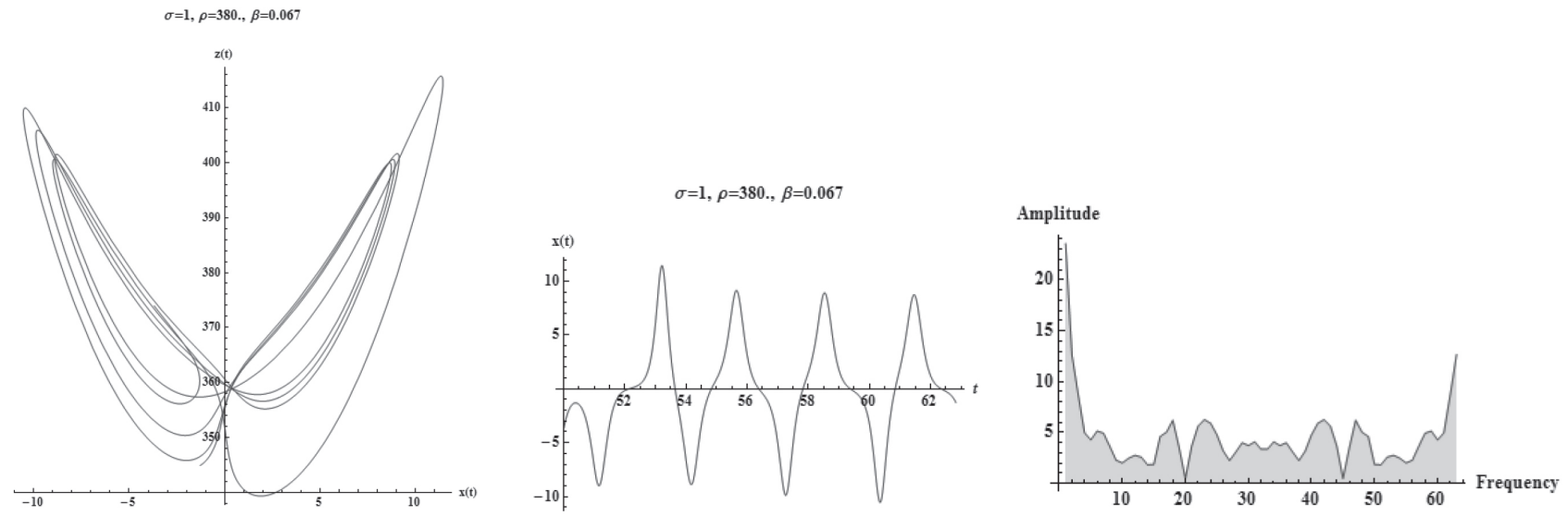

Figure 10. 2D phase "portrait", time evolution and Fourier transform for $r=380, b=0.067$.

The limit cycle appears through an inverse sub-harmonic cascade and it loses its stability due to a new chaotic „window" intermitent transition.

We show in Fig. 11 the Lyapunov exponent map for $\sigma=1$ of the biological Lorenz system (the light points coordinates represent the values pair $(x, y)=(b, r)$ for which the chaotic regime probability is high).

All of these denote the complexity of extracellular vesicles convection in haptotaxis with hydrodynamical dissipation.

\section{Results and Discussion}

The main results of the present paper are as follows:

1) we build the first Lorenz model for extracellular vesicles migration;

2) in Sukumuran and Seifert (2001) and similar other references, it has been shown that EVs under shear flow close to a substrate behave in very much the same way as that found in two dimensional simulations, so we are confident that the $2 \mathrm{D}$ assumptions captures the essential features of the 3D EVs;

3) different control parameter values for the Lorenz system can create shape distributions similar to the cordonal appearance of fingerprints (see Fig. 12A), or complex skin tissue tiles like scale appendages in the amphibian covering (see Fig. 12B); Fig. 12 - Bénard-Rayleigh model patterns representative for biological instances: A) for fingerprint like distribution of skin cells, B) for fish or amphibian scales;

4) the biological thought experiment equivalent to Bénard's experiment, involving a fluid layer of extracellular vesicles adherent to an extracellular matrix, in a haptotactic gradient can be checked experimentally today to a high degree of accuracy. We think that suitable test systems would be the embryological ones (i.e. the development 


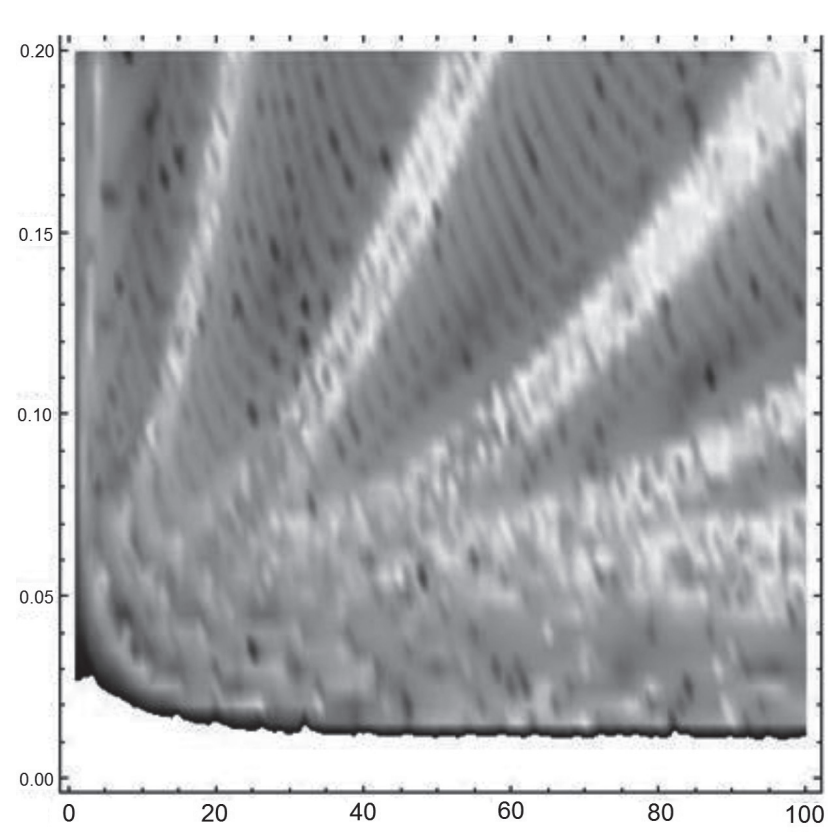

Figure 11. Lyapunov exponent map for $\sigma=1$ of the biological Lorenz system.

of branched vessels in membranes - avian eggshell membranes, serous membranes of the peritoneal cavity; or the budding development of lung alveoli, or of fingerprints), and, similar, inflammatory ones (i.e. the emergence of neoangionetic vessels driven by inflammatory proximities) - all of which apparently start as point like spots displayed in a comb-like appearance along a rectilinear or arched origin;

5) we analyze the problem of EVs migration in haptotaxis, though most of the reasoning applies to chemotaxis (migration of cells biased towards a gradient of diffusible MDEs) as well as to a variety of driving forces - all of which include the possibility to specify an active parameter value within the model.

The resulted system of equations exhibits complex behavior, hard to control, the two occurring convective rolls: either going in one direction, or in the opposite one - means patterning the EVs spreading. Such a model shows the profoundly nonlinear property of the biological systems. In such context the collective type effect becomes dominant with respect to the individual one.

Acknowledgements. This work was supported by the strategic grant POSDRU/159/1.5/S/133652, Project "Integrated support system for improving doctoral and post-doctoral research quality in Romania and for advocating the role of science in society" co-financed by the European Social Found within the Sectorial Operational Program Human Resources Development 2007-2013.
A
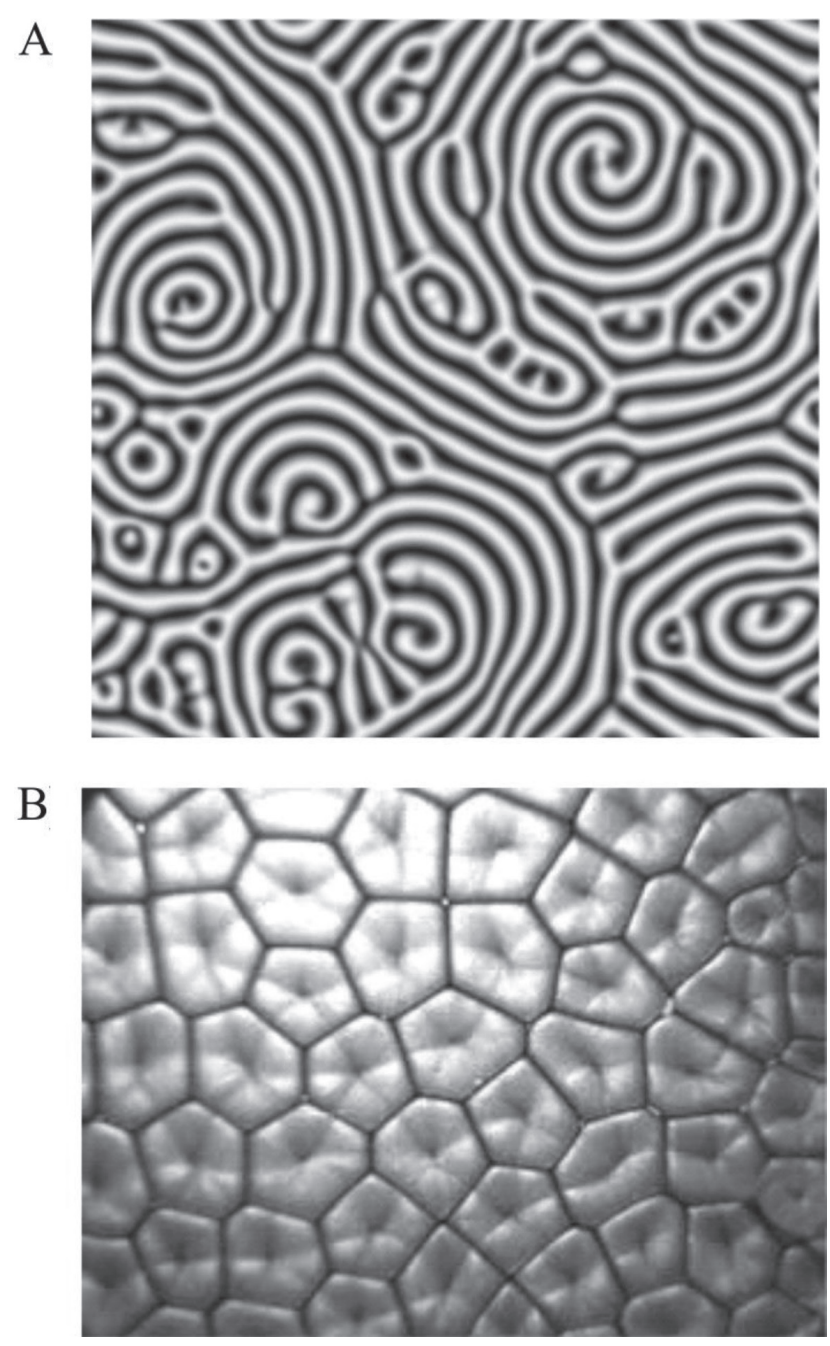

Figure 12. Bénard-Rayleigh model patterns representative for biological instances: fingerprint like distribution of skin cells (A) and fish or amphibian scales (B).

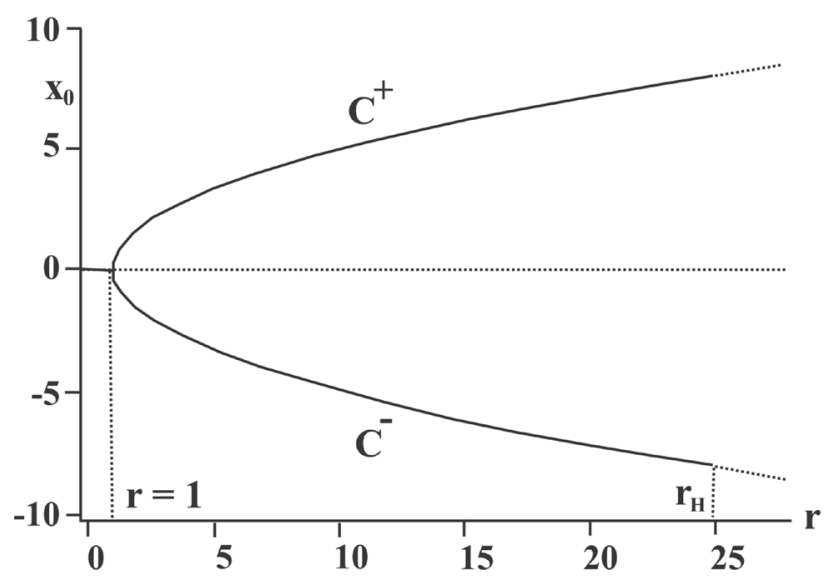

Figure 13. A pitchfork bifurcation with two fixed points, $C^{+}$and $C^{-}$ that correspond with the patterns from the Benard experiment. 


\section{Appendix A - The standard Lorenz system and its prop- erties}

Let us consider the evolution equations of the Lorenz system (Lorenz 1963; Jackson 1992):

$$
\begin{aligned}
& \dot{X}=\sigma(Y-X) \\
& \dot{Y}=-X Z+r X-Y \\
& \dot{Z}=X Y-b Z
\end{aligned}
$$

We note that our biological Lorenz system $(30 a-c)$ is reduced to the above written standard Lorenz system in the case that Pr from Eq. (20a) is identical with the parameter $\sigma$, i.e. $\operatorname{Pr} \equiv \sigma$.

The system $(\mathrm{A} .1 \mathrm{a}-\mathrm{C})$ is dissipative due to the fact that the divergence (for details see (Jackson 1992)

$$
\nabla \cdot F=\frac{\partial F_{x}}{\partial x}+\frac{\partial F_{y}}{\partial y}+\frac{\partial F_{z}}{\partial z}=-\sigma-1-b<0
$$

\section{because $b>0$.}

Therefore the phase volume exponentially decreases with time, as much as the system tends toward the attractor. For any value of the control parameter $r$ the system (A.1a-c) allows for a fixed point the origin,

$$
X_{0}=Y_{0}=Z_{0}=0
$$

The characteristic equation can be written as:

$$
\left|\begin{array}{ccc}
-\sigma-\omega & \sigma & 0 \\
r-Z_{0} & -1-\omega & -X_{0} \\
Y_{0} & X_{0} & -b-\omega
\end{array}\right|=0
$$

For the fixed point (A.3), this equation takes the form

$$
\left|\begin{array}{ccc}
-\sigma-\omega & \sigma & 0 \\
r & -1-\omega & 0 \\
0 & 0 & -b-\omega
\end{array}\right|=0
$$

from which we find

$$
(b+\omega)\left[\omega^{2}+(1+\sigma) \omega-\sigma(r-1)\right]=0
$$

From the fact that the parameters $b, r$, and $\sigma$ are positive results that the first eigenvalue, $\omega_{1}=-b$ is negative for any parameters values. The other two eigenvalues, $\omega_{2}$ and $\omega_{3}$, satisfy the relations:

$$
\left\{\begin{array}{l}
\omega_{2}+\omega_{3}=-(1+\sigma)<0 \\
\omega_{2} \omega_{3}=-\sigma(r-1)
\end{array}\right.
$$

In agreement with $(\mathrm{A} .7 \mathrm{a}, \mathrm{b})$, if $0<r<1$, then the sum of the two eigenvalues is negative and their product is positive. Therefore all the eigenvalues are negative and the origin is a stable node. Also in agreement with (A.7a,b), for $r>1$, the origin becomes unstable, and two new fixed points that correspond with the patterns from the Benard experiment, $C^{+}$ and $C^{-}$, appear in a pitchfork bifurcation (Jackson 1992).

$$
\begin{gathered}
\left(C^{+}\right)\left\{\begin{array}{l}
X_{0}=Y_{0}=\sqrt{b(r-1)} \\
Z_{0}=r-1
\end{array}\right. \\
\left(C^{-}\right)\left\{\begin{array}{l}
X_{0}=Y_{0}=-\sqrt{b(r-1)} \\
Z_{0}=r-1
\end{array}\right.
\end{gathered}
$$

Let us study the stability of these points. By replacing the adequate values for the $C^{+}$branch in Eq. (A.4), the characteristic equation becomes:

$$
\left|\begin{array}{ccc}
-\sigma-\omega & \sigma & 0 \\
1 & -1-\omega & -\sqrt{b(r-1)} \\
\sqrt{b(r-1)} & \sqrt{b(r-1)} & -b-\omega
\end{array}\right|=0
$$

From this it results that

$$
\omega^{3}+\omega^{2}(b+\sigma+1)+\omega b(\sigma+r)+2 b \sigma(r-1)=0
$$

If the fixed points (A.8) will be subjected to a Hopf bifurcation, then, for a value of the control parameter $r_{\mathrm{H}}>1$, two conjugated complex purely imaginary eigenvalues will exist at the bifurcation point. By replacing $\omega=i \beta$ in (A.10) we obtain

$$
-i \beta^{3}-\beta^{2}(b+\sigma+1)+i \beta b(\sigma+r)+2 b \sigma(r-1)=0
$$

By separating the real from the imaginary part in (A.11), the following system results

$$
\begin{aligned}
& -\beta^{3}+\beta b(\sigma+r)=0 \\
& -\beta^{2}(b+\sigma+1)+2 b \sigma(r-1)=0
\end{aligned}
$$

From Eq. (A.12a) it results that $\beta^{2}=b(\sigma+r)$. By replacing this value in Eq. (A.12b), we conclude that the Hopf bifurcation takes place at

$$
r_{H}=\sigma \frac{b+\sigma+3}{\sigma-b-1}
$$

For this value of the control parameter, the two fixed points $\mathrm{C}^{+}$and $C^{-}$lose their stability in a subcriticall Hopf bifurcation. Beyond the bifurcation point, all of the periodical orbits are 
unstable and the system displays a chaotic behavior. Further increasing the control parameter $r$ value, an intricate succession of chaotic regimes follows, with certain periodicity windows. The limit cycle appears through an inverse subharmonic cascade and loses its stability through a tranzition by intermittences towards a new chaotic window. All of these facts are presented in detail in (Sparrow 1982; Jackson 1992).

\section{References}

Abkarian M., Lartigue C., Viallat A. (2001): Motion of phospholipidic vesicles along an inclined plane. Sliding and rolling. Phys. Rev. E 63, 041906 http://dx.doi.org/10.1103/PhysRevE.63.041906

Abkarian M., Lartigue C., Viallat A. (2002): Tank treading and unbonding of deformable vesicles in shear flow. Determination of the lift force. Phys. Rev. Lett. 88, 068103 http://dx.doi.org/10.1103/PhysRevLett.88.068103

Biben T., Misbah C. (2002): An advected-field method for deformable entities under flow. Euro. Phys. J. B 29, 311-316 http://dx.doi.org/10.1140/epjb/e2002-00307-6

Bousinessq J. (1871): Théorie de l'intumescence liquide, applelée onde solitaire ou de translation, se propageant dans un canal rectangulaire. Comptes Rendus de l'Academie des Sciences 72, 755-759 (in French)

Brochard F., Lennon J.-F. (1975): Frequency spectrum of the flicker phenomenon in erythrocytes, J. Phys. France 36, 1035-1047 http://dx.doi.org/10.1051/jphys:0197500360110103500

Cantat I., Misbah C. (1999a): Dynamics and similarity laws for adhering vesicles in haptotaxis. Phys. Rev. Lett. 83, 235-238 http://dx.doi.org/10.1103/PhysRevLett.83.235

Cantat I., Misbah C. (1999b): Lift force and dynamical unbinding of adhering vesicles under shear flow. Phys. Rev. Lett. 83, 880-883 http://dx.doi.org/10.1103/PhysRevLett.83.880

Cantat I., Kassner K., Misbah C. (2003): Vesicles in haptotaxis with hydrodynamical dissipation. Eur. Phys. J. E 10, 175-189 http://dx.doi.org/10.1140/epje/e2003-00022-1

Durand I., Jönson P., Misbah C., Valance A., Kassner K. (1997): Adhesion-induced vesicle propulsion. Phys. Rev. E 56, 3776 http://dx.doi.org/10.1103/PhysRevE.56.R3776

Fritz J. (1991): Partial Differential Equations (4th ed.). Springer, Berlin

Jackson E. A. (1992): Perspectives on Nonlinear Dynamics (vol $1+2)$, Cambridge University Press, Cambridge

Johnson R.S. (1997): A modern introduction to the mathematical theory of water waves. Cambridge Texts in Applied Mathematics 19. Cambridge University Press, Cambridge
http://dx.doi.org/10.1017/CBO9780511624056

Helfrich W. (1973): Elastic properties of lipid bilayers-theory and possible experiments. Z. Naturforsch. C. 28, 693-703

Kern N., Fourcade B. (1999): Vesicles in linearly forced motion. Europhys. Lett. 46, 262-267 http://dx.doi.org/10.1209/epl/11999-00254-x

Kraus M., Wintz W., Seifert U., Lipowsky R. (1996): Fluid vesicles in shear flow. Phys. Rev. Lett. 77, 3685-3688 http://dx.doi.org/10.1103/PhysRevLett.77.3685

Landau L. D., Lifshitz E. M. (1987): Fluid Mechanics. Volume 6 of Course of Theoretical Physics, 2nd English Edition, Revised. Pergamon Books Ltd

Lipowsky R., Sackmann E. (1995): Structure and Dynamics of Membranes. Handbook of Biological Physics. Elsevier, NorthHolland

Lorenz E. N. (1963): Deterministic non-periodic flow. Journal of Atmospheric Sciences 20, 130-141 http://dx.doi.org/10.1175/1520-0469(1963)020<0130:DNF$>2.0$.CO; 2

Lorenz E. N. (2005): Designing Chaotic Models. Journal of the Atmospheric Sciences 62, 5, 1574-1587 http://dx.doi.org/10.1175/JAS3430.1

Lortz B., Simon R., Nardi J., Sackmann E. (2000): Weakly adhering vesicles in shear flow, Tanktreading and anomalous lift force. Europhys. Lett. 51, 468 http://dx.doi.org/10.1209/epl/i2000-00517-6

Manneville P. (1992): Structures dissipatives, chaos et turbulence. Collection Alea Saclay, Paris

Nardi J., Bruinsma R., Sackmann E. (1999): Vesicles as osmotic motors. Phys. Rev. Lett. 82, 5168-5171 http://dx.doi.org/10.1103/PhysRevLett.82.5168

Prost J., Bruinsma R. (1996): Shape fluctuations of active membranes. Europhys. Lett. 33, 321-326 http://dx.doi.org/10.1209/epl/i1996-00340-1

Schuster H. G. (1995): Deterministic Chaos: An Introduction. 3rd edition. VCH, Weinheim

Seifert U. (1999): Hydrodynamic lift on bound vesicles. Phys. Rev. Lett. 83, 876-879 http://dx.doi.org/10.1103/PhysRevLett.83.876

Sparrow C. (1982): The Lorenz Equations: Bifurcations, Chaos, and Strange Attractors. Springer, Berlin http://dx.doi.org/10.1007/978-1-4612-5767-7

Sukumaran S., Seifert U. (2001): Influence of shear flow on vesicles near a wall: A numerical study. Phys. Rev. E 64, 011916 http://dx.doi.org/10.1103/PhysRevE.64.011916

Received: July 7, 2015

Final version accepted: November 18, 2015

First published online: April 5, 2016 